\title{
Re: Wernicke's Encephalopathy after Laparoscopic Roux-en-Y Gastric Bypass: A Misdiagnosed Complication
}

\author{
Antonio Iannelli - Anne Sophie Schneck • \\ Pietro Addeo • Jean Gugenheim
}

Published online: 11 June 2010

(C) Springer Science+Business Media, LLC 2010

Sir,

We read with interest the comments of Dr. Zhang and colleagues to the case report on Wernicke-Korsakoff (WK) that we recently published on the Obesity Surgery Journal [1].

Dr. Zhang raises the problem of the differential diagnosis of WK syndrome including the extrapontine myelinolysis (EPM) and polyneuropathy resulting from thiamine deficiency. They suggest the differential diagnosis of EPM because of the severe dehydration that the patient experienced before being referred at our institution and because serum levels of the thiamine were not determined at the moment of admission.

Although the differential diagnosis of EPM must be kept in mind, this is a well-known pitfall associated with rehydration in patients with severe hyponatremia. Indeed, any physician is supposed to be aware of this potential complication of rehydration and be able to deal with it. For this reason, we assumed that the patient resuscitation was made according to the state of art to prevent EPM.

Furthermore, although we did not measure the blood thiamine levels, the clinical findings, magnetic resonance imaging evidence, and clinical response to treatment strongly suggest the diagnosis of WK due to thiamine deficiency as in one of the references quoted by Zhang and colleagues [2,3].

We agree that polyneuropathy due to thiamine deficiency should have been considered as part of the clinical picture and further investigated with electrophysiological studies. However, treatment would have remained the same [2, 3].

We stress that surgeons should be aware of the need to supplement bariatric patients with thiamine systematically and promptly in the setting of persistent vomiting before the development of this life-threatening complication.

\section{References}

1. Iannelli A, Addeo P, Novellas S, et al. Wernicke's encephalopathy after laparoscopic Roux-en-Y gastric bypass: a misdiagnosed complication. Obes Surg. 2010 Mar 1. doi:10.1007/s11695-010-0116-0.

2. Cirignotta F, Manconi M, Mondini S, et al. Wernicke-Korsakoff encephalopathy and polyneuropathy after gastroplasty for morbid obesity. Arch Neurol. 2000;57:1356-9.

3. Sechi GP, Serra A. Wernicke's encephalopathy: new clinical settings and recent advances in diagnosis and management. Lancet Neurol. 2007;6:442-55.

A. Iannelli $(\bowtie) \cdot$ A. S. Schneck $\cdot$ P. Addeo $\cdot$ J. Gugenheim Service de Chirurgie Digestive et Centre de Transplantation Hépatique, Pôle Digestif, Hôpital Archet 2,

151 Route Saint Antoine de Ginestière, BP 3079 , 06202 Nice Cedex3, France

e-mail: antonio_iannelli@hotmail.com

A. Iannelli $\cdot$ A. S. Schneck $\cdot$ P. Addeo $\cdot$ J. Gugenheim

Université de Nice Sophia-Antipolis,

06107 Nice, France 